\title{
A Case for the Coexistence of Heterogeneous Wireless Networks
}

\author{
Xinyu Zhang and Kang G. Shin \\ Department of Electrical Engineering and Computer Science \\ The University of Michigan \\ \{xyzhang, kgshin\}@eecs.umich.edu
}

\begin{abstract}
As the ISM spectrum becomes crowded by various network devices, such as WiFi and ZigBee, the coexistence between them poses a critical challenge, due to their heterogeneous MAC/PHY layers. Recent measurement studies have shown moderate to high WiFi traffic to severely impair coexisting ZigBee's performance. We propose a new mechanism, called cooperative busy tone (CBT), that exploits the inherent cooperation among ZigBee nodes to harmonize their coexistence with WiFi devices. CBT employs a separate ZigBee node to emit a busy-tone concurrently with the desired data transmission, thereby improving the visibility of ZigBee devices to $\mathrm{WiFi}$. It adopts an innovative way to concurrently schedule the busy-tone and a data packet without causing interference between them. To optimize CBT, we establish a theoretical framework that models the performance of coexisting ZigBee and WiFi networks, and analyzes the impact of key design parameters in CBT. Our analytical results and testbed implementation demonstrate CBT's significant throughput improvement over the legacy ZigBee protocol, with negligible performance loss to $\mathrm{WiFi}$.
\end{abstract}

\section{Categories and Subject Descriptors}

C.2.1 [Computer-Communication Networks]: Network Architecture and Design; Network Protocols

\section{General Terms}

Design, Performance, Reliability, Theory

\section{Keywords}

Coexistence of heterogeneous networks, ZigBee (802.15.4) and WiFi (802.11), busy-tone

\section{INTRODUCTION}

The past decade has witnessed the proliferation of wireless devices (e.g., ZigBee and $\mathrm{WiFi}$ ) running on the ISM band, and the coexistence between them becomes increasingly critical. As an interference avoidance mechanism, CSMA becomes ineffective for coordinating heterogeneous devices, which may adopt asynchronous time slots, different scheduling modes (e.g., TDMA vs. CSMA), disparate transmission/interference

Permission to make digital or hard copies of all or part of this work for personal or classroom use is granted without fee provided that copies are not made or distributed for profit or commercial advantage and that copies bear this notice and the full citation on the first page. To copy otherwise, to republish, to post on servers or to redistribute to lists, requires prior specific permission and/or a fee.

S3'11, September 19, 2011, Las Vegas, Nevada, USA.

Copyright 2011 ACM 978-1-4503-0XXX-X/11/09 ...\$10.00.

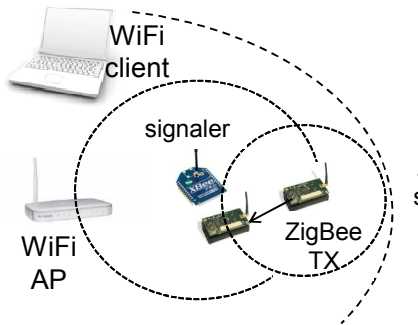

(a)

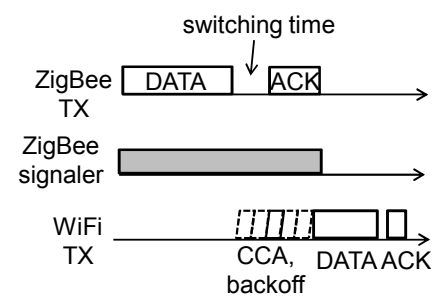

(b)
Figure 1: The principle behind CBT: (a) spatial domain: allowing WiFi to indirectly sense weak ZigBee signals. (b) temporal domain: avoiding WiFi preempting in the $\mathrm{rx} / \mathrm{tx}$ switching time of $\mathrm{ZigBee}$.

ranges, and incompatible communication mechanisms. For example, recent measurement studies have shown ZigBee's performance to be severely degraded in the presence of moderate to high WiFi traffic. In an enterprise WiFi WLAN and a co-located 90-node ZigBee WPAN for building energy management, more than a half of the ZigBee links were observed to suffer connection loss during peak hours due to WiFi interference [7]. The harmful coexistence has also been observed in previous small- to medium-scale experimental deployments $[4,5,8]$.

A further microscopic study [9] has revealed the root cause of such harmful coexistence. First, ZigBee's transmit power is $20 \mathrm{~dB}$ lower than WiFi's, yielding a smaller spatial footprint and hence, its poor visibility to WiFi. The ZigBee's MAC-layer time slot is also 16 times longer, and it can easily be preempted by $\mathrm{WiFi}$ in the middle of a $\mathrm{rx} / \mathrm{tx}$ transition (e.g., sensing-to-transmission or data-to-ACK transition), thus causing collision, even if they can sense each other. In addition, besides CSMA, ZigBee allows for TDMA mode, which operates without carrier sensing, and may arbitrarily collide with an ongoing WiFi transmission.

Based on the above observations, we propose a new mechanism, called Cooperative Busy Tone (CBT), to facilitate ZigBee's coexistence with WiFi. Unlike the traditional CSMA that relies on a data packet as an implicit carrier signal (busy-tone), CBT assigns a separate ZigBee node called signaler, as a proxy to perform the carrier signaling, i.e., sending a busy-tone packet concurrently with the data packet. The signaler may have a higher power than the ZigBee transmitter, thus allowing the WiFi nodes to sense the ZigBee's presence indirectly by detecting the busy-tone (Fig. 1(a)). The busy-tone continues throughout the data and ACK roundtrip, thus preventing the WiFi's preemption in the $\mathrm{rx} / \mathrm{tx}$ switching gap (Fig. 1(b)). The core component in CBT is an innovative way to concurrently schedule the busy-tone and a data packet without causing interference between them.

To understand the performance of CBT, we have developed an analytical framework that models a heterogeneous network where ZigBee and WiFi coexist. Our analysis quan- 


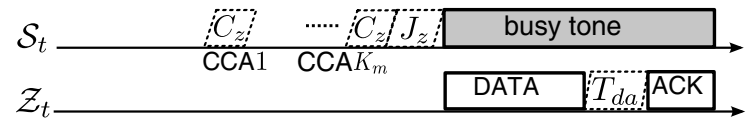

Figure 2: The TDMA scheduler in CBT. $C_{z}(128 \mu s)$ denotes the CCA duration; $J_{z}(192 \mu s)$ the CCA-to-tx switching time (or channel switching time); $T_{d a}$ the data-to-ACK switching time.

tifies the network performance, with and without running CBT. We have also prototyped CBT based on TinyOS and the GNURadio library [1]. Our experiments on the MicaZ motes and USRP2 [3] software radio platform further corroborate the feasibility and effectiveness of CBT.

\section{DESIGN AND ANALYSIS}

\subsection{Frequency Flip}

A key challenge to CBT is that the signaler's busy-tone must occur concurrently with the data transmission (without interrupting it). To overcome this difficulty, we design a frequency flip mechanism that separates the busy-tone from the data transmission in frequency domain, yet ensures $\mathrm{WiFi}$ to sense the presence of ZigBee transmission. The frequency flip exploits the inherent spectrum heterogeneity between ZigBee and WiFi. On the $2.4 \mathrm{GHz}$ ISM band, each WiFi channel overlaps with 4 orthogonal ZigBee channels. When running the frequency flip, the signaler hops to an adjacent channel before starting the busy tone, and hops back to the original channel immediately after the busy tone is transmitted. This way, CBT ensures the busy tone is orthogonal to the data packet, but still overlaps with the WiFi channel and can cause it to defer transmission.

\subsection{Busy Tone Scheduler}

A ZigBee WPAN assigns a coordinator node to schedule a mixture of TDMA and CSMA slots periodically. CBT maintains this legacy protocol, but requires the signaler to dispatch the busy tone at an appropriate time, so as to reduce the WiFi preemptions of ZigBee transmissions, and to minimize the potential influence on WiFi performance. Due to space constraint, we only discuss how CBT protects ZigBee's TDMA packets. A detailed introduction to the CSMA mode is available in [10].

Fig. 2(a) illustrates the procedure to send a TDMA packet. In CBT, the coordinator announces the start time of each slot to the signaler as well as the target clients. Before each slot, the signaler performs CCA (for at most $K_{m}$ times) in order to avoid interfering with $\mathrm{WiFi}$. At the first idle CCA, it runs the frequency flip, switches to the adjacent channel, and starts the busy tone immediately. The busy tone lasts from the first idle CCA to the end of the TDMA slot, to protect both the data and ACK packets from WiFi interruption.

We establish a theoretical framework to analyze the performance of CBT in comparison with the legacy ZigBee. Our analysis pinpoints a key design parameter, $K_{m}$, that specifies how early the signaler should start the busy-tone.

Temporal Collision Probability. We start with the temporal collision probability, i.e., probability that WiFi and ZigBee's packets overlap in time. We assume a single ZigBee and WiFi link coexist, and their packet arrival follows a Poisson process. Besides the notations in Fig. 2, we further use $\lambda_{z}$ to denote the packet arrival rate of $\mathcal{Z}_{t}$ (and $\lambda_{w}$ for $\mathcal{W}_{t}$, the WiFi transmitter). Let $\tau_{z}$ be $\mathcal{Z}_{t}$ 's data

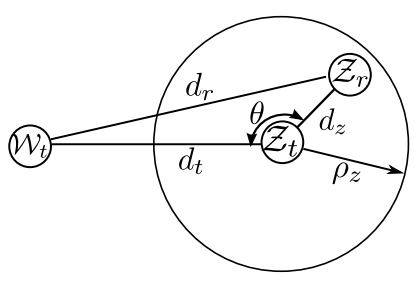

Figure 3: A model for analyzing the spatial collision probability.

packet duration, and assume $\tau_{z} \ll \lambda_{z}^{-1}$ (since $\mathcal{Z}_{t}$ runs low duty-cycle applications). Further, let $\beta_{w}$ be the life-cycle of a WiFi packet when $\mathcal{W}_{t}$ senses an idle channel, assuming $\beta_{w}>C_{z}$ and WiFi traffic is non-saturated (i.e., $\beta_{w}<\lambda_{w}^{-1}$ ). Then, we have the following results (which can be straightforwardly derived from the analysis in [10]):

Proposition 1 When running legacy ZigBee, the temporal collision probability for $\mathcal{Z}_{t}$ 's data packets is approximately $\lambda_{w} \beta_{w}$ when $\mathcal{W}_{t}$ can sense $\mathcal{Z}_{t}$, and $1-e^{-\lambda_{w}\left(\beta_{w}+\tau_{z}\right)}$ otherwise. When running CBT, the busy-tone fails with probability $\lambda_{w} \beta_{w} C^{K_{m}-1}$, where $C=1-\frac{C_{z}}{\beta_{w}}<1$. Hence, CBT scales down the collision probability by at least a factor that increases exponentially with $K_{m}$.

Spatial Collision Probability. Temporal collision does not necessarily cause packet loss. Due to the capture effect, the desired packet can still be decoded if its power is sufficiently higher than the interference. Such opportunities depend on the relative location of $\mathrm{WiFi}$ and ZigBee.

Consider a randomly located ZigBee link and WiFi interferer, as shown in Fig. 3. We assume $\theta \sim U(0,2 \pi)$, and $d_{z} \sim U\left(0, \rho_{z}\right)$, where $\rho_{z}$ is the maximum distance between ZigBee transmitter/receiver (i.e., $\mathcal{Z}_{t}$ and $\mathcal{Z}_{r}$ ). Since ZigBee and WiFi have similar receiver sensitivity (around $-86 \mathrm{dBm}[2,6]$ ), we assume $\mathcal{W}_{t}$ and $\mathcal{Z}_{t}$ have the same carrier sensing threshold. We use a generic pathloss model, where the ratio between received power and transmit power is $c d_{z}^{-\alpha}$. The constant $c$ depends on the propagation characteristics, and $\alpha$ is the environment dependent pathloss exponent. Denote $C_{a}$ as the capture threshold, $\Lambda_{z}$ and $\Lambda_{w}$ as the transmit power of $\mathcal{Z}_{t}$ and $\mathcal{W}_{t}$, respectively, then $\mathcal{Z}_{r}$ fails to decode the packet if:

$$
\frac{\Lambda_{z} c d_{z}^{-\alpha}}{\Lambda_{w} c d_{r}^{-\alpha}} \leq C_{a} \text {, or equivalently, } \frac{d_{r}^{2}}{d_{z}^{2}} \leq\left(C_{a} \frac{\Lambda_{w}}{\Lambda_{z}}\right)^{\frac{2}{\alpha}}
$$

The key to our analysis is to derive the probability that the two link distances satisfy the above collision condition, which we denote as $I_{e}$, or spatial collision probability. Based on an approximation to the cosine rule [10], we have:

$$
I_{e}=\max \left\{0,1-d_{t} c_{1}^{-\frac{1}{2}} \rho_{z}^{-1}\right\}, \text { and } c_{1} \triangleq\left(C_{a} \frac{\Lambda_{w}}{\Lambda_{z}}\right)^{\frac{2}{\alpha}}-1
$$

Obviously, when $\rho_{z}$, transmit power and capture threshold are fixed, $I_{e}$ decreases linearly with $d_{t}$, i.e., $\mathcal{W}_{t}$ 's interference effect diminishes linearly as it moves away from $\mathcal{Z}_{t}$.

\subsection{Signaler Configuration}

When using a dedicated signaler, its location and transmit power must be carefully configured so that the busy tone may be sensed by the potential $\mathrm{WiFi}$ interferers randomly located near the ZigBee WPAN or moving around. In CBT, a single signaler is placed near the coordinator. When the signaler's power level is sufficiently high, all nodes in the 


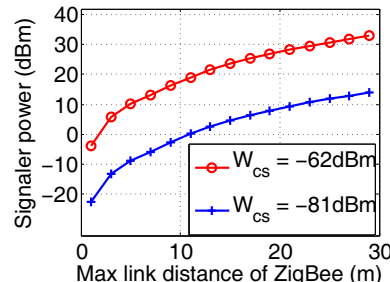

(a)

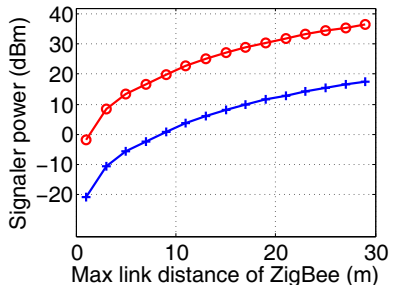

(b)
Figure 5: Required signaling power to ensure the WPAN is sensible by WiFi: (a) uplink (b) downlink.

WPAN are likely to be equally protected. We verify this intuition based on insights from a simple analytical model.

Let $\Lambda_{s}$ be the transmit power of the signaler, and $W_{c s}$ the carrier sensing threshold of WiFi. Suppose the signal's path-loss function (in normal scale) is $L(d)$, where $d$ is the distance. Then we have (proof available in [9]):

Proposition 2 To protect the entire WPAN from arbitrarily located interferers, $\Lambda_{s}$ should satisfy:

$$
\begin{array}{r}
\Lambda_{s} \geq \frac{\Lambda_{w}}{5 \Lambda_{z}} W_{c s} L\left(\rho_{z}\right) C_{a} \\
\Lambda_{s} \geq \frac{\Lambda_{w}}{5 \Lambda_{z}} W_{c s} L\left(\rho_{z}+M_{w r}\right) C_{a}
\end{array}
$$

for protecting uplink and downlink packets, respectively. $M_{w r}$ is the maximum distance between the coordinator and potential interferer that can cause packet loss, which satisfies:

$$
L\left(M_{w r}\right)=L\left(\rho_{z}\right) C_{a} \Gamma_{w}\left(5 \Gamma_{z}\right)^{-1}
$$

From the results, we see that the required signaling power increases with the ZigBee link distance $\rho_{z}$, and with the WiFi carrier sensing threshold $W_{c s}$. Higher signaling power is needed to protect downlink packets, since the signaler is located farther away from the clients than from the coordinator. Note that a maximum distance $M_{w r}$ exists, because the WiFi transmitter can cause packet loss (hence becoming a potential interferer) only if its signal power exceeds the desired ZigBee packet power by the capture threshold $C_{a}$.

To gain a concrete understanding of Proposition 2, we configure the parameters to their typical values (converted to $d B$ scale): $\Lambda_{w}=15 \mathrm{dBm}, \Lambda_{z}=0 \mathrm{dBm}, C_{a}=10 \mathrm{~dB}$. We use am empirical propagation model proposed in IEEE 802.15.3 [9], and consider two typical carrier sensing thresholds used by WiFi [9]: $W_{c s}=-81 \mathrm{dBm}$ and $W_{c s}=-62 \mathrm{dBm}$.

Fig. 5 shows the required power that makes the signaler visible to any potential interferers co-located with the WPAN. Even when $\mathcal{W}_{t}$ is insensitive $\left(W_{c s}=-62 d B m\right)$, a normal ZigBee signaler with $0 \mathrm{dBm}$ power can be effective for short range WPANs with $\rho_{z}<2 \mathrm{~m}$. When $\rho_{z}>10 \mathrm{~m}$, the signaler needs at least $20 \mathrm{dBm}$ power to prevent interference from WiFi transmitters. In such cases, a dedicated signaler that has power comparable to WiFi's is required.

\section{EXPERIMENTAL RESULTS}

Combining the analysis for temporal and spatial collision, we can analyze how ZigBee is affected by a WiFi transmitter at an arbitrary location. We fix $\Lambda_{z}$ to $-10 \mathrm{dBm}$, WiFi traffic load ( $\lambda_{w}$ normalized by the maximum packet arrival rate) to a medium value 0.36 , and then calculate the probability of packet loss, i.e., both spatial and temporal collision occurs. The results (Fig. 4) shows that for legacy ZigBee, the loss probability is a piecewise linear function of $d_{t}$, according to whether $\mathcal{W}_{t}$ and $\mathcal{Z}_{t}$ can sense each other. When $\mathcal{W}_{t}$ can sense $\mathcal{Z}_{t}$, the loss rate is relatively low (below $41 \%$ ).
However, when $\mathcal{W}_{t}$ cannot sense $\mathcal{Z}_{t}$, the collision rate first increases dramatically (to above $68 \%$ ), and then decreases gradually as $\mathcal{W}_{t}$ moves away from $\mathcal{Z}_{t}$. Using the signaler, CBT extends the range where $\mathcal{Z}_{t}$ is visible to $\mathcal{W}_{t}$ and prevents WiFi preemption in the time domain. These two advantages together bring the loss rate to below $7 \%$.

We have also implemented CBT on the TinyOS/MICAz and GNURadio/USRP platforms (see [9] for more details about our implementation). The main findings from our testbed experiments are summarized as follows.

- $\mathrm{CBT}$ reduces the WiFi-caused collision by $42 \%$ to $90 \%$, depending on the WiFi airtime usage, relative location, scheduling mode, etc.. Compared to the retransmission mechanism, it reduces not only packet loss but also the average packet delay by up to $63 \%$.

- CBT can improve the performance of an entire WPAN with multiple ZigBee nodes, which coexist with randomly located WiFi transmitters. Compared to an ideal adaptive channel allocation protocol, it achieves a similar long-term throughput, but a $59.4 \%$ less disruption time.

- With a dedicated high-power signaler, CBT saves energy by $73.2 \%$ to $83.3 \%$ by protecting ZigBee's TDMA packets from interruption.

- WiFi performance is degraded when running CBT or ZigBee in case of high airtime usage (60\%), but unaffected under low duty-cycle traffic (below 10\%).

\section{REFERENCES}

[1] The GNU Software Radio. http://gnuradio.org/trac/wiki.

[2] Wireless LAN Medium Access Control (MAC) and Physical Layer (PHY) Specifications. IEEE Std. 802.11, 2007.

[3] Ettus Research LLC. Universal Software Radio Peripheral (USRP). http://www.ettus.com/.

[4] R. Gummadi, H. Balakrishnan, and S. Seshan. Metronome: Coordinating Spectrum Sharing in Heterogeneous Wireless Networks. In First International Workshop on Communication Systems and Networks (COMSNETS), 2009.

[5] J. Huang, G. Xing, G. Zhou, and R. Zhou. Beyond Co-existence: Exploiting WiFi White Space for ZigBee Performance Assurance. In Proc. of IEEE ICNP, 2010.

[6] IEEE-SA Standard Board. IEEE Standard Part 15.4: Wireless Medium Access Control (MAC) and Physical Layer (PHY) Specifications for Low-Rate Wireless Personal Area Networks (LR-WPANs), 2003.

[7] C.-J. M. Liang, N. B. Priyantha, J. Liu, and A. Terzis. Surviving Wi-Fi Interference in Low Power ZigBee Networks. In Proc. of ACM SenSys, 2010.

[8] S. Pollin, I. Tan, B. Hodge, C. Chun, and A. Bahai. Harmful Coexistence Between 802.15.4 and 802.11: A Measurement-based Study. In ICST CrownCom, 2008.

[9] X. Zhang and K. G. Shin. Cooperative Carrier Signaling: Harmonizing Coexisting WPAN and WLAN Devices. http:

//www. eecs. umich. edu/ xyzhang/papers/CCS.pdf. Submitted to IEEE Transactions on Networking, 2011

[10] X. Zhang and K. G. Shin. Enabling Coexistence of Heterogeneous Wireless Systems: Case for ZigBee and WiFi. In Proc. of ACM MobiHoc, 2011. 